\title{
Direct Mechanisms, Menus and Latent Contracts
}

\author{
Gwenaël Piaser ${ }^{1,2}$ \\ ${ }^{1}$ IPAG Business School, Paris, France \\ ${ }^{2}$ Luxembourg School of Finance, Luxembourg, Luxembourg \\ E-mail: gwenael.piaser@uni.lu \\ Received February 17, 2010; revised March 20, 2010; accepted March 30, 2010
}

\begin{abstract}
In common agency games, one cannot characterize all equilibria by considering only direct mechanisms. In an attempt to overcome this difficulty, Peters [1] and Martimort and Stole [2] identified a class of indirect mechanisms (namely, menus) which are able to characterize every equilibrium. Unfortunately, menus are difficult to handle, and several methodologies have been proposed in the literature. Here, it is shown that, even if authors consider menus rather than simpler mechanisms, many equilibria described in the literature could have been characterized by direct incentive compatible mechanisms. Use of more sophisticated mechanisms was not necessary in these cases.
\end{abstract}

Keywords: Common Agency, Revelation Principle, Delegation Principle, Direct Mechanisms, Menus, Latent Contracts

\section{Introduction}

The restriction to direct incentive compatible mechanisms is a cornerstone of contract theory. It provides a simple and elegant method for characterizing arbitrary equilibria in any principal-agent model, even with very complex communication between the players. Because of its tractability, the principal-agent model has been very successful, and it has revitalized many economic fields: Regulation, redistribution, insurance and others. ${ }^{1}$ Multiagent games have provided the basis for auction theory and the theory of the provision of public goods.

Unfortunately, the restriction to direct incentive compatible mechanisms causes some loss of generality in multi-principal games. Intuitively, simple contracts fail to be general because the structure of the game involves endogenous information. For a principal, relevant information includes not only the type of the agent (for example his/her willingness to pay in a case of a duopoly) but also the message that the agent sends to other principals; the message sent sets a particular agreement between a principal and the agent, which could modify the agent's willingness to pay for the products of other principals.

A strategy for overcoming this limitation is to give up the concept of "direct mechanism" or any of its generalizations, and consider the Taxation Principle. This principle was introduced by Hammond [4], Guesnerie [5]

\footnotetext{
${ }^{1}$ See Laffont and Martimort [3] for a complete survey.
}

and Rochet [6], and states that there is no loss of generality in considering menus, or nonlinear prices. Peters [1] and Martimort and Stole [2] show that an equivalent of the Taxation Principle (they call it Delegation Principle) makes it possible to characterize any equilibrium of any common agency game. The problem with this approach is that the concept of menu is large for common agency games, and, even if it simplifies the game, equilibria remain hard to characterize. To reach tractable problems, other ad hoc assumptions are added to restrict the menu set.

The present paper does not question the validity of the differing further assumptions made in the literature. We welcome assumptions (differentiability or continuity) if they allow ready characterization of equilibria in this class of games. The cost of these assumptions is probably a loss of generality. ${ }^{2}$ Nevertheless, the author does not believe that restrictions invalidate the results obtained with menus. The methodologies used to find a fixed-point in common agency games in which menus are allowed are criticized. The present paper shows that, in almost all models of the common agency literature, equilibria characterized by menus could have been characterized by direct mechanisms. The basic intuition is that menus can characterize a large set of equilibria because a principal, by using a menu, can create sophisticated rewards.

\footnotetext{
${ }^{2}$ In common agency games, some equilibria may be sustained by discontinuous menus; see Laffont and Tirole [7] chap. 17.
} 
Given menus, it might seem that analysis of common agency games is simply a matter of computation. Unfortunately, though the use of menus may be helpful in this class of game, it does not permit ready characterization of equilibria. Below, it is argued that common methodologies used in the literature characterize only a restricted set of pure strategy equilibria. Let us now consider the "latent contract" concept, which gives insight into the main result.

The next section presents a basic common agency model. Section 3 defines direct mechanisms and menus. Section 4 introduces the concept of latent contracts. In section 5 some examples are presented from the literature. Section 6 sets out conclusions.

\section{The Model}

Consider a scenario in which there are a number of principals (indexed by $i \in\{1, \ldots, n\}=N$ ) contracting with one agent (denoted by index 0 ). The agent's type is drawn from a compact set $\Theta$ having a probability distribution $F($.) that is common knowledge. The principal $i$ also makes an action: He has to decide which allocation $y_{i} \in Y_{i}$ to implement. The implemented allocation is observable and contractible. This means that a principal can write a contract which specifies his chosen allocation. Let us make the stronger assumption that a principal is not able to contract on a probability distribution over $Y_{i}$. The payoff to principal $i$ is represented by the von Neumann-Morgenstern utility function

$$
V_{i}: Y_{1} \times \ldots \times Y \times \Theta \rightarrow \mathfrak{R}
$$

and for the agent the payoff is represented by the function

$$
U_{i}: Y_{1} \times \ldots \times Y \times \Theta \rightarrow \mathfrak{R}
$$

Preferences could be more general; the restriction to von Neumann-Morgenstern utility functions is not critical for any of the following results, but merely makes the model simpler. Moreover, applications in the literature invariably consider this class of preferences. The principals compete through mechanisms. Each principal's mechanism is a map from $M_{i}$ to $Y_{i}$, where $M_{i}$ is the message space. Each set $M_{i}$ (for any principal $i$ ) is compact, and each map $\sigma_{i}$ is measurable. For clarity, we require any mechanisms $\sigma_{i}$ to be such that the image set $\sigma_{i}\left(M_{i}\right)$ is a compact set. We denote by $\Sigma_{i}$ the set of all available map $\sigma_{i}$ to principal $i$ when his message space is $M_{i}$. We denote by $M=\prod_{i \in N} M_{i}$ the collection of the chosen message space, and $\Sigma$ is the collection of $\Sigma_{i}$. Denote by $\sigma_{i}\left(m_{i}\right)$ the decision $y_{i}$ in $Y_{i}$ that the agent gets by sending the message $m_{i}$ to the principal $i$. We explicitly assume that the rule $\sigma_{i}($.$) is enforceable. Once a principal has announced a$ mechanism, he commits himself to respect his own rule; if he receives a message $m_{i}$, he cannot choose a decision different from $\sigma_{i}\left(m_{i}\right)$. Finally, since the sets $\sigma_{i}\left(M_{i}\right)$ are compact, it follows that

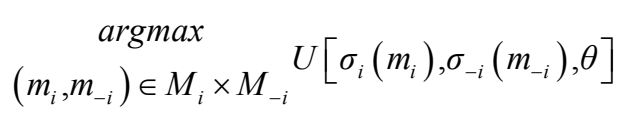

The agent's pure strategy is to choose a message $m_{i} \in M_{i}$ for each principal. Hence, a pure strategy for the agent is a map $\sigma_{0}: \Theta \times \Sigma \rightarrow M$. We denote by $\Sigma_{0}$ the collection of all these possible pure strategies and by $\tilde{\sigma}_{0 i}$ the message sent to principal $i$.

Given the type set $\Theta$, the common agency game is the array:

$$
\Gamma_{M}=\left\{\Theta,\left(\Sigma_{i}\right)_{i \in N}, \Sigma_{0}, U(., \theta),\left(V_{i}(., \theta)\right)_{i \in N}, F(.)\right\}
$$

\section{Direct Mechanisms and Menus}

In the game $\Gamma_{M}$ we have made only made standard assumptions concerning the sets $M_{i}$. The message spaces may be quite complex. We can simplify the game by considering direct mechanisms. For each principal the message space $M_{i}$ is given, and coincides with the agent's type space $\Theta$. We thereby restrict the strategy spaces of the principals.

The strategy of principal $i$ is the map $\tilde{\sigma}_{i}: \Theta \rightarrow Y_{i}$; we let $\tilde{\Sigma}_{i}$ be the strategy space for principal $i$, and $\tilde{\Sigma}$ be the collection of all such strategy profiles. The strategy of the agent is then a map $\tilde{\sigma}_{0}$, and $\tilde{\Sigma}_{0}$ denotes the collection of all such maps by $\tilde{\sigma}_{0 i}$ the message sent to principal $i$.

Given $\Theta$, the common agency game induced by direct mechanism is the array:

$$
\tilde{\Gamma}_{\Theta}=\left\{\Theta,\left(\tilde{\Sigma}_{i}\right)_{i \in N}, \tilde{\Sigma}_{0}, U(., \theta),\left(V_{i}(., \theta)\right)_{i \in N}, F(.)\right\}
$$

Direct mechanisms have an obvious appeal- the message spaces are simple and given. But, to be useful, we need more than a simplification of the message space. To apply the traditional principal-agent methodology, we need also a definiton of the concept of incentive compatibility in our context.

Definition 1 A collection of strategies $\left(\left(\tilde{\sigma}_{i}^{*}\right)_{i \in N}, \tilde{\sigma}_{0}^{*}\right)$ is an incentive compatible equilibrium of the game $\tilde{\Gamma}_{\Theta}$ if it satisfies the following two conditions: 


$$
\forall \theta \in \Theta, \quad \tilde{\sigma}_{0}^{*}\left(\left(\tilde{\sigma}_{i}^{*}\right)_{i \in N}, \theta\right)=(\theta, \ldots, \theta)
$$

and

$$
\begin{aligned}
& \forall i \in N, \forall \theta \in \Theta, \\
& \text { if } \hat{\sigma}_{i} \in \tilde{\Sigma}_{i} \text { is such that } \tilde{\sigma}_{0 i}^{*}\left(\hat{\sigma}_{i}, \tilde{\sigma}_{-i}, \theta\right)=\theta
\end{aligned}
$$

then $\hat{\sigma}_{i}$ is not a profitable deviation.

When is it possible to restrict attention to direct incentive compatible mechanisms? In other words, is it always the case that, for any equilibrium of the game $\Gamma_{M}$, there exists a incentive compatible equilibrium of the game $\tilde{\Gamma}_{\Theta}$ such that the two equilibria are outcome equivalent? For common agency games this is not so (see, for instance, Peck [8], Martimort and Stole [2]). In games with multiple principals, equilibria may exist whose outcomes cannot be supported in equilibrium in the corresponding direct mechanism game.

Peters [1] and Martimort and Stole [2] show that even if one cannot restrict attention to direct incentive compatible mechanisms, a modified version of the Taxation Principle applies. This principle states that, without loss of generality:

1) One can restrict the set of message space and consider the sets of all compact subsets of $Y_{i}$ rather than $M_{i}$.

2) The map $\sigma_{i}$ is the identity over the chosen subset of $Y_{i}$.

Given $Y=\prod_{i \in N} Y$, the common agency game induced by menu is the array

$$
\Gamma_{Y}=\left\{\Theta,\left(Z_{i}\right)_{i \in N}, \bar{\Sigma}_{0}, U(., \theta),\left(V_{i}(., \theta)\right)_{i \in N}, F(.)\right\}
$$

where $\bar{\Sigma}_{0}$ is defined as $\Sigma_{0}$ and $\bar{\sigma}_{0 i}^{*}$ as $\sigma_{0 i}^{*}$. Where $Z_{i}$ denotes the set of all compact subsets of $Y_{i}$, and $T_{i}$ is a generic element of $Z_{i}$. We will use the obvious notation $T=\prod_{i \in N} T_{i}$.

Theorem 1 The three following statements can be established:

1) For every equilibrium $\left(\sigma^{*}, \sigma_{0}^{*}\right)$ of the game $\Gamma_{M}$ there exists an outcome equivalent equilibrium $\left(T^{*}, \bar{\sigma}_{0}^{*}\right)$ of the game $\Gamma_{Y}$.

2) For any equilibrium $\left(T^{*}, \bar{\sigma}_{0}^{*}\right)$ of the game $\Gamma_{Y}$ there exists an outcome equivalent equilibrium $\left(\sigma^{*}, \sigma_{0}^{*}\right)$ of the game $\Gamma_{M}$.

3) For any incentive compatible pure strategy equilibrium $\left(\tilde{\sigma}^{*}, \tilde{\sigma}_{0}^{*}\right)$ of the game $\tilde{\Gamma}_{\Theta}$, there exists a pure strategy equilibrium of the game $\Gamma_{M}$ such that the two equilibria are outcome equivalent.

The two first statements have been shown by Peters [1] and Martimort and Stole [2]. The third is a result from Peters [9]. The last statement of the theorem is very general, and it does not rely on any assumption about the action space available to the agent. It applies to all common agency models in the literature to date.

\section{Latent Contracts}

This section demonstrates how "latent contracts" can help to characterize a larger set of equilibria. ${ }^{3}$ By latent contract or or latent decision is meant any decision reachable by the agent but never implemented at equilibrium, whatever the type of the agent. For example, if a principal uses a incentive compatible direct mechanism, this mechanism does not involve any "latent contract". This former mechanism is a map from the type set $\Theta$ to the decision set $Y_{i}$, denoted $\tilde{\sigma}_{i}$. By definition, for any decision $\tilde{y}_{i}$ in the image set $\tilde{\sigma}_{i}(\Theta)$, there is a type $\theta \in \Theta$ such that $\tilde{\sigma}_{i}(\theta)=\tilde{y}_{i}$.

Definition 2 We say that a menu $T_{i}$ contains latent decisions if, given the strategies of the other players $T_{-i}$ and $\bar{\sigma}_{0}^{*}$,

$$
\exists y_{i} \in T_{i}, \forall \theta \in \Theta, \bar{\sigma}_{0 i}^{*} \neq y_{i}
$$

From this definition, we can reach the following theorem:

Theorem 2 Consider the game $\Gamma_{Y}$ and a pure strategy equilibrium. If principals offer menus without latent decisions, then there exists an output equivalent incentive compatible equilibrium in the direct mechanisms game $\Gamma_{\Theta}$.

Proof Consider an equilibrium $\left(T_{i}^{*}, T_{-i}^{*}, \sigma_{0}^{*}\right)$ of the game $\Gamma_{Y}$. We wish to construct an output equivalent equilibrium in the game $\Gamma_{\Theta}$. By assumption, menus $\left(T_{i}, T_{-i}\right)$ do not involve latent decisions. One can consider the agent's equilibrium best reply $\bar{\sigma}_{0}^{*}$, which is, for every collection of menu $T \in Z$, a function from set $T \times \Theta$ to the set $T$. We can construct unambiguously the following direct mechanisms denoted $\bar{\sigma}_{i}^{*}$ :

$$
\forall i \in N, \quad \forall \theta \in \Theta, \quad \tilde{\sigma}_{i}^{*}(\theta)=\bar{\sigma}_{0 i}^{*}\left(T_{i}, T_{-i}, \theta\right)
$$

where the mapping $\bar{\sigma}_{0 i}^{*}$ is defined as above. We have

${ }^{3}$ Latent contracts were introduced by Hellwig [10], and the concept is widely used in the literature on foundations of competitive equilibrium; see for example Bisin et al. [11]. 
constructed the strategies $\left(\tilde{\sigma}_{i}^{*}, \tilde{\sigma}_{-i}^{*}\right) \in \tilde{\Sigma}$ of the principals in the game $\Gamma_{\Theta}$. Let us construct the agent's best reply, denoted $\tilde{\sigma}_{0}$. For all $\tilde{\sigma} \in \tilde{\Sigma}$ and for all $\theta \in \Theta$, we denote by $\tilde{\sigma}_{i}(\Theta)$ the image set of the mapping $\sigma_{i}$. Define the best reply mapping of the agent as follows:

$$
\forall \tilde{\sigma} \in \tilde{\Sigma}, \forall \theta \in \Theta, \quad \tilde{\sigma}_{0}^{*}(\tilde{\sigma}, \theta)=\bar{\sigma}_{0}^{*}\left(\tilde{\sigma}_{i}(\Theta), \theta\right)
$$

For all collections of direct mechanism $\tilde{\sigma} \in \tilde{\Sigma}$, we have

$$
\tilde{\sigma}_{0}^{*}(\tilde{\sigma}, \theta)=\underset{\left(h_{1}, h_{2}, \ldots, h_{n}\right) \in \Theta^{N}}{\operatorname{argmax}} U\left(\left(\tilde{\sigma}_{i}\left(h_{i}\right)\right)_{i \in N}, \theta\right)
$$

\section{Suppose not:}

$$
\begin{aligned}
& \exists\left(h_{1}, h_{2}, \ldots, h_{n}\right) \in \Theta^{N}, \quad \exists \theta \in \Theta, \\
& U\left(\left(\tilde{\sigma}_{i}\left(h_{i}\right)\right)_{i \in N}, \theta\right)>U\left(\left(\tilde{\sigma}_{i}\left(\tilde{\sigma}_{0 i}^{*}(\sigma, \theta)\right)_{i \in N}\right), \theta\right)
\end{aligned}
$$

By construction,

$U\left(\left(\tilde{\sigma}_{i}\left(\tilde{\sigma}_{0 i}^{*}(\tilde{\sigma}, \theta)\right)\right)_{i \in N}, \theta\right)=U\left(\left(\bar{\sigma}_{0 i}^{*}\left(\tilde{\sigma}_{i}(\Theta)\right)\right)_{i \in N}, \theta\right)$. Consequently,

$$
U\left(\left(\bar{\sigma}_{0 i}^{*}\left(\tilde{\sigma}_{i}(\Theta)\right)\right)_{i \in N}, \theta\right)>U\left(\left(\tilde{\sigma}_{i}\left(h_{i}\right)\right)_{i \in N}, \theta\right)
$$

Since by construction we have $\forall i \in N, \quad \tilde{\sigma}\left(h_{i}\right) \in \tilde{\sigma}_{i}(\Theta)$, we generate a contradiction.

Moreover, we have:

$$
\forall \theta \in \Theta, \tilde{\sigma}_{0}^{*}(\tilde{\sigma}, \theta)=(\theta, \ldots, \theta)
$$

because, by definition for all $\theta \in \Theta$ and for every principal $i, \tilde{\sigma}_{i}^{*}(\theta)=\bar{\sigma}_{0 i}^{*}\left(T^{*}, \theta\right)$. Hence the candidate equilibrium is incentive compatible.

Supppose that principals play $\tilde{\sigma}^{*}$, and principal $i$ deviates toward $\tilde{\sigma}_{i} \in \tilde{\Sigma}_{i}$ (all other players keep their strategies). The agent's bast play is then $\tilde{\sigma}_{0}^{*}\left(\tilde{\sigma}_{i}, \tilde{\sigma}_{-i}^{*}, \theta\right)$. The "no latent decision assumption" implies that

$$
\forall i \in N, \forall y_{i} \in T_{i}^{*}, \quad \exists \theta \in \Theta \text { such that } \bar{\sigma}_{0 i}^{*}\left(T_{i}^{*}, \theta\right)=y_{i}
$$

Hence

$$
\forall i \in N, \quad \tilde{\sigma}_{i}^{*}(\Theta)=T_{i}^{*}
$$

Using the definition of $\tilde{\sigma}_{0}^{*}$, we can state that

$$
\begin{aligned}
& \forall j \in N, \forall i \in N, \quad \forall \tilde{\sigma}_{i} \in \tilde{\Sigma}_{i}, \\
& \tilde{\sigma}_{0 j}^{*}\left(\tilde{\sigma}_{i}, \tilde{\sigma}_{-i}^{*}, \theta\right)=\bar{\sigma}_{0 j}^{*}\left(\sigma_{i}(\Theta), T_{-i}^{*}, \theta\right)
\end{aligned}
$$

Under the "no latent decision" condition:

$$
\begin{aligned}
& \forall i \in N, \quad \forall y_{i} \in T_{i}^{*}, \quad \exists h \in \Theta, \\
& \text { such that } \tilde{\sigma}_{i}^{*}(h)=y_{i}
\end{aligned}
$$

Hence, for all $\theta \in \Theta$, there exists $\left(h_{i}(\theta), h_{i}(\theta)\right) \in \Theta^{N}$ such that:

$$
\begin{aligned}
& \left(\tilde{\sigma}_{i}\left(h_{i}(\theta)\right),\left(\tilde{\sigma}_{j}^{*}\left(h_{j}(\theta)\right)\right)_{j \in N \backslash\{i\}}\right) \\
& =\left(\bar{\sigma}_{0 i}^{*}\left(\sigma_{i}(\Theta), T_{-i}^{*}, \theta\right),\left(\bar{\sigma}_{0 j}^{*}\left(\sigma_{i}(\Theta), T_{-i}^{*}, \theta\right)\right)_{j \in N \backslash\{i\}}\right)
\end{aligned}
$$

Moreover, by definition $\left(h_{i}(\theta), h_{i}(\theta)\right)=\tilde{\sigma}_{0 j}^{*}\left(\tilde{\sigma}_{i}, \tilde{\sigma}_{-i}^{*}, \theta\right)$.

Suppose that for principal $i$ the deviation is strictly profitable:

$$
\begin{aligned}
& \int V_{i}\left(\left(\tilde{\sigma}_{0 j}^{*}\left(\tilde{\sigma}_{i}, \tilde{\sigma}_{-i}^{*}, \theta\right)\right)_{j \in N}, \theta\right) d F(\theta) \\
& >\int V_{i}\left(\left(\tilde{\sigma}_{0 j}^{*}\left(\tilde{\sigma}_{i}^{*}, \tilde{\sigma}_{-i}^{*}, \theta\right)\right)_{j \in N}, \theta\right) d F(\theta)
\end{aligned}
$$

Equation (19) can now be rewritten as

$$
\begin{aligned}
& \int V_{i}\left(\left(\bar{\sigma}_{0 j}^{*}\left(\tilde{\sigma}_{i}(\Theta), T_{-i}^{*}, \theta\right)\right)_{j \in N}, \theta\right) d F(\theta) \\
& >\int V_{i}\left(\left(\bar{\sigma}_{0 j}^{*}\left(T_{i}^{*}, T_{-i}^{*}, \theta\right)\right)_{j \in N}, \theta\right) d F(\theta)
\end{aligned}
$$

which is a contradiction. We conclude that $\left(\tilde{\sigma}_{i}^{*}, \tilde{\sigma}_{-i}^{*}, \tilde{\sigma}_{0}^{*}\right)$ is an equilibrium of the game $\tilde{\Gamma}_{\Theta}$. By construction, this equilibrium is output equivalent to the equilibrium $\left(T_{i}^{*}, T_{i}^{*}, \bar{\sigma}_{0}^{*}\right)$.

Consider now the following example:

Example 1 Each principal $(i=1,2)$ must make a decision ( $y_{i}^{1}$ or $y_{i}^{2}$ with $i=1,2$ ). The corresponding payoffs are given by the following matrix:

\begin{tabular}{ccc}
\hline & $y_{2}^{1}$ & $y_{2}^{2}$ \\
\hline$y_{1}^{1}$ & $(2,2,3)$ & $(0,3,1)$ \\
$y_{1}^{2}$ & $(1,0,1)$ & $(1,1,2)$ \\
\hline
\end{tabular}

where the first element in each cell refers to the payoff of Principal 1, the second element to the payoff of Principal 2 , and the last element to the agent's payoff.

If we consider that principals are using direct mechanisms (which are take-it or leave-it offers since information is complete), there is only one pure strategy equilibrium: Principal 1 plays $y_{1}^{2}$ and principal 2 plays $y_{1}^{2}$. Agent's payoffs are not relevant, since the the agent plays no role.

If principals are allowed to use menus, so that they offer subsets of $\left\{y_{i}^{1}, y_{i}^{2}\right\}$, then there are two pure strategy 
equilibria. In the first equilibrium, principal 1 offers the degenerated menu $\left\{y_{1}^{2}\right\}$ and the principal 2 offers the menu $\left\{y_{2}^{2}\right\}$. In the second equilibrium, principal 1 offers the menu $\left\{y_{1}^{1}, y_{1}^{2}\right\}$ and principal 2 offers the menu $\left\{y_{2}^{1}, y_{2}^{2}\right\}$. The agent chooses $y_{1}^{1}$ from principal 1 and $y_{2}^{1}$ from principal 2 . The outcome $\left(y_{1}^{1}, y_{2}^{1}\right)$ is finally implemented. The outcome $\left(y_{1}^{1}, y_{2}^{1}\right)$ cannot be supported by an equilibrium if principals use only direct mechanisms. If principal 1 offers $\left\{y_{1}^{1}\right\}$, the direct mechanism $\left\{y_{2}^{1}\right\}$ is not the best reply for principal 2 . He gets more by offering $\left\{y_{2}^{2}\right\}$. The outcome $\left(y_{1}^{1}, y_{2}^{1}\right)$ can be implemented because menus $\left\{y_{1}^{1}, y_{1}^{2}\right\}$ and $\left\{y_{2}^{1}, y_{2}^{2}\right\}$ embed latent decisions: $y_{1}^{2}$ and $y_{2}^{2}$ are not chosen by the agent, but they are crucial because they prevent deviations.

Let us consider a second example.

Example 2 The type of the agent is $\theta_{1}$ with probability $p_{1}=1 / 2$, and $\theta_{2}$ with probability $1-p_{1}$. Payoffs are given by the following matrices:

\begin{tabular}{ccc}
\hline & $\theta_{1}$ & \\
\hline & $y_{2}^{1}$ & $y_{2}^{2}$ \\
$y_{1}^{1}$ & $(2,2,3)$ & $(0,3,1)$ \\
$y_{1}^{2}$ & $(1,0,1)$ & $(1,1,2)$ \\
\hline & $\theta_{2}$ & \\
\hline & $y_{2}^{1}$ & $y_{2}^{2}$ \\
$y_{1}^{1}$ & $(0,1,2)$ & $(1,0,1)$ \\
$y_{1}^{2}$ & $(0,3,1)$ & $(2,2,3)$ \\
\hline
\end{tabular}

This game has an equilibrium in the menu game. Each principal proposes the menu $\left\{y_{i}^{1}, y_{i}^{2}\right\}, i=1,2$; the agent chooses $\left(y_{1}^{1}, y_{2}^{1}\right)$ if his type is $\theta_{1}$, and chooses $\left(y_{1}^{2}, y_{2}^{2}\right)$ if his type is $\theta_{2}$. Since the set of possible menus is very small, we can check that for principal 1 no deviations (which are the singletons) are profitable. The same holds for principal 2. The outcome can also be supported as an equilibrium in the direct mechanism game. For principal 1 the former strategy can be reproduced in the following way: he plays $y_{1}^{1}$ if the agent sends the message $\theta_{1}$, and plays $y_{1}^{2}$ if the agent announce $\theta_{2}$. Principal 2 plays the same strategy $\left(y_{2}^{1}\right.$ if $\theta_{1}$ and $y_{2}^{2}$ if $\theta_{2}$ ). The agent can reach any cell by misreporting his type. By analogy with the menu game, it is best for the agent to announce his real type. Using a similar argument, one can check that the strategies described are also best replies for the principals.

In the preceding example, the menus $\left\{y_{1}^{1}, y_{1}^{2}\right\}$ and $\left\{y_{2}^{1}, y_{2}^{2}\right\}$ do not embed latent decisions. For principal 1, $y_{1}^{1}$ (resp. $y_{1}^{2}$ ) is chosen when the agent's type is $\theta_{1}$ (resp. $\theta_{2}$ ). Similarly, for principal 2, item $y_{2}^{1}$ is chosen if the agent is of type $\theta_{1}$, and $y_{2}^{2}$ is chosen when the type is $\theta_{2}$. Menus do not embed latent decisions, so that the equilibrium can be sustained by direct mechanisms. Moreover, at equilibrium, the agent reveals his true type.

Consider a last example taken from the literature.

Example 3 (Biais and Mariotti [12]) There are two principals (indexed by $i=1,2$ ), and their decision spaces are $Y_{1}=Y_{2}=Y=\mathfrak{R}^{+} \times[0,1]$. A generic decision is denoted by $(t, q)$. The two principals have the same utility functions $\theta q-t$, where $\theta$ is the information of the agent, $\theta \in[0,1]$. The distribution function of $\theta$ over $[0,1]$ is denoted by $F$. Agent preferences are represented by the utility function $t-\lambda \theta q$, where the variable $\lambda \in(0,1)$; this is common knowledge. The agent is constrained to accept contracts $\left(t_{1}, q_{1}\right)$ and $\left(t_{2}, q_{2}\right)$ such that $q_{1}+q_{2} \leq 1$. Assume that $E(\theta)<\lambda$. Then an equilibrium exists in which each principal offers the menu $M=\{(t, q) \in Y$ such that $q=t E(\theta)\}$. If the two principals offer this menu, the agent (whatever his type) will choose the allocation $q=1$ and $t=E(\theta)$. If one principal deviates, and offers a unique contract $\left(t^{\prime}, q^{\prime}\right)$, then

1) If $\left(t^{\prime}, q^{\prime}\right)$ is below the line $t=q E(\theta)$, the agent will accept this contract and the deviating principal makes zero profit, as he did at equilibrium. The deviation is not profitable.

2) If $\left(t^{\prime}, q^{\prime}\right)$ is above the line $t=q E(\theta)$, the agent will always accept the contract offered. Whatever his type, the agent will buy a quantity $q=1 \quad q^{\prime}$ from the other principal. Since $t^{\prime} / q^{\prime}<E(\theta)$, the deviating principal makes losses.

This argument can easily extended to any kind of menu. If we now look at the best direct mechanism against the menu $M$, it is obvious that the degenerated mechanism gives the contract $(E(\theta), 1)$ to every type. 
It is also clear that, if one principal plays that mechanism, it is not the best reply for the other principal to play that same mechanism. He should offer the contract $\left(t^{\prime}, q^{\prime}\right)$, where $t^{\prime}=\lambda q^{\prime}$. This contract is accepted by the agent only when his type is $\theta=1$, in which case it provides positive profit to the principal. The equilibrium characterized by Biais and Mariotti [12] is efficient, so that if we ignore it, we may reach wrong conclusions.

\section{Applied Common Agency Models}

Let us now focus on examples taken from the literature.

To characterize equilibria in the set of menus is not a trivial exercise. Martimort [13] and Martimort and Stole [2] have introduced a sophisticated methodology.

1) They consider that principal $i$ uses direct mechanisms $\tilde{\sigma}_{1}^{*}$ to reply to the menus $T_{-i}^{*}$ and to the agent's strategy.

2) From the best direct mechanism, one can deduce a menu.

3) If this is done for every principal, and if each principal is playing the menu derived from the best direct mechanism, we finally obtain an equilibrium.

At equilibrium, menus do not involve latent decisions; each item is chosen by some agent. This method provides no gain over the traditional method; any equilibrium characterized using this methodology can be characterized by the simple use of direct mechanisms. Here is an example that shows how this methodology fails to characterize any equilibrium of a common agency game.

Example 4 The type of the agent is $\theta_{1}$ with probability $p_{1}=1 / 2$, and $\theta_{2}$ with probability $1-p_{1}$. Payoffs are given by the following matrices:

\begin{tabular}{ccc}
\hline & $\theta_{1}$ & \\
\hline & $y_{2}^{1}$ & $y_{2}^{2}$ \\
$y_{1}^{1}$ & $(2,2,3)$ & $(0,3,1)$ \\
$y_{1}^{2}$ & $(1,0,1)$ & $(1,1,4)$ \\
\hline & $\theta_{2}$ & \\
\hline & $y_{2}^{1}$ & $y_{2}^{2}$ \\
$y_{1}^{1}$ & $(4,2,2)$ & $(2,3,0)$ \\
$y_{1}^{2}$ & $(1,0,1)$ & $(1,1,5)$ \\
\hline
\end{tabular}

This common agency game has one pure strategy equilibrium. The first principal (P1) plays the menu $\left\{y_{1}^{1}, y_{1}^{2}\right\}$ get an utility of 3 and the principal 2 plays the menu $\left\{y_{2}^{1}\right\}$, and gets an expected utility of 2. Clearly, the first principal has no profitable deviation (he gets his maximum payoff). The second principal has two possible deviations in the menu game: $\left\{y_{2}^{2}\right\}$ and $\left\{y_{2}^{1}, y_{2}^{2}\right\}$. If the second principal plays $\left\{y_{2}^{2}\right\}$ or the menu $\left\{y_{2}^{1}, y_{2}^{2}\right\}$ the agent will always choose the item $\left\{y_{1}^{2}\right\}$ in principal's 1 menu. Hence, deviating gives to principal 2 an expected utility of 1 .

Using the Martimort-Stole algorithm we cannot characterize the equilibrium described above. If principal 2 is playing the menu $\left\{y_{2}^{1}\right\}$, the unique best reply of principal 1 is to play the direct mechanism (with incentive compatible revelation of type):

$$
\tilde{\sigma}_{1}\left(\theta_{1}\right)=\tilde{\sigma}_{1}\left(\theta_{2}\right)=y_{1}^{1}
$$

Playing this mechanism is equivalent to playing the menu $\left\{y_{1}^{1}\right\}$. The best reply to the menu $\left\{y_{1}^{1}\right\}$ for principal 2 (in the set of direct mechanisms) is to play the mechanism

$$
\tilde{\sigma}_{2}\left(\theta_{1}\right)=\tilde{\sigma}_{2}\left(\theta_{2}\right)=y_{2}^{2}
$$

or equivalently the menu $\left\{y_{2}^{2}\right\}$, and not the menu $\left\{y_{1}^{2}\right\}$. By construction, menus characterized by the Martimort-Stole algorithm do not embed latent decisions; they support equilibria which can also be supported by direct mechanisms.

This methodology has been used, explicitly or implicitly, in several papers: Biais et al. [14] Calzolari [15], Laffont and Pouyet [16], Martimort and Stole [17] and Khalil et al. [18], and in several other unpublished papers: Olsen and Osmundsen [19], Diaw and Pouyet [20] or Calzolari and Scarpa [21]. These authors are able to characterize regular and realistic equilibria. Nevertheless, these equilibria could have been characterized using the standard methods of mechanisms design. Moreover, the authors may not succeed in characterizing all of the equilibria of the communication game.

When Martimort and Stole [17] consider a complete information version of their game (i.e., when $[\Theta]=1$; roughly speaking, when their model is qualitatively similar to our first example) by using menus, they are able to characterize equilibria that could not have been characterized by direct mechanisms. (Direct mechanisms are take-it or leave-it offers in that case.) They do not then use the methodology presented above. Hence for complete information games, their analysis of their model is invaluable. $^{4}$

Martimort [22] proposes an original methodology. He proposes focusing on direct mechanisms, and extends the

${ }^{4}$ They use the term "singleton contracts" instead of take-it or leave-it offers. 
type set: the agent can report a type belonging to the set $\tilde{\Theta}$, with $\tilde{\Theta} \subset \Theta, \tilde{\Theta} \neq \Theta$. At equilibrium, whatever his type is, the agent is reporting his true type, but the fact that he can report $\tilde{\theta} \in \tilde{\Theta} \backslash \Theta$, (a type which does not exist) and get an outcome that cannot be reached if he reports any $\theta \in \Theta$, extends his possible strategies. The message $\tilde{\theta}$ is never sent at equlibrium, but the possibility of reporting an "absurd type" makes some deviations of the other principals unprofitable, as in examples 1 and 3. Clearly, this methodology is able to characterize equilibria that cannot be characterized if we restrict our attention to direct mechanisms. Sending the message $\tilde{\theta}$ is clearly a "latent decision".

Let us reconsider our last example.

The equilibrium can be also characterized in the following way. The first principal plays the direct mechanism

$$
\begin{gathered}
\tilde{\sigma}_{1}:\left\{\theta_{1}, \theta_{2}, \tilde{\theta}\right\} \rightarrow\left\{y_{1}^{1}, y_{1}^{2}\right\} \\
\begin{cases}\tilde{\sigma}_{1}\left(\theta_{1}\right)=y_{1}^{1} \\
\tilde{\sigma}_{1}\left(\theta_{2}\right)=y_{1}^{1} \\
\tilde{\sigma}_{1}(t \theta)=y_{1}^{2}\end{cases}
\end{gathered}
$$

where $\tilde{\theta}$ is an absurd type.

The second principal plays the direct mechanism

$$
\begin{aligned}
& \tilde{\sigma}_{2}:\left\{\theta_{1}, \theta_{2}, \tilde{\theta}\right\} \rightarrow\left\{y_{2}^{1}, y_{2}^{2}\right\} \\
& \left\{\begin{array}{l}
\tilde{\sigma}_{2}\left(\theta_{1}\right)=y_{2}^{1} \\
\tilde{\sigma}_{2}\left(\theta_{2}\right)=y_{2}^{1}
\end{array}\right.
\end{aligned}
$$

The direct mechanisms $\tilde{\sigma}_{1}$ and $\tilde{\sigma}_{2}$ constitute an equilibrium. The best strategy for the agent is to reveal his type. (We do not describe the agent's strategy, as it is very long and is not necessary.) The first principal has no profitable strategy; he gets his maximum payoff in each state of nature. For the second principal there are many possible direct mechanisms. But the second principal cannot get a payoff greater than 2 : in every state of nature, if $y_{2}^{2}$ is implemented, the agent will report the type $\tilde{\theta}$ to the first principal 2 (whatever his real type) and the second principal will get a payoff of value 1 . Thus, principal 2 has no profitable deviations. If principal 1 plays the direct mechanism

Thus, principal 2 has no profitable deviations. If principal 1 plays the direct mechanism

$$
\begin{gathered}
\tilde{\sigma}_{1}:\left\{\theta_{1}, \theta_{2}\right\} \rightarrow\left\{y_{1}^{2}, y_{1}^{2}\right\} \\
\left\{\begin{array}{l}
\tilde{\sigma}_{1}\left(\theta_{1}\right)=y_{1}^{1} \\
\tilde{\sigma}_{1}\left(\theta_{2}\right)=y_{1}^{1}
\end{array}\right.
\end{gathered}
$$

then the second principal has a profitable deviation; playing the mechanism

$$
\left\{\begin{array}{l}
\tilde{\sigma}_{2}\left(\theta_{1}\right)=y_{2}^{2} \\
\tilde{\sigma}_{2}\left(\theta_{2}\right)=y_{2}^{2}
\end{array}\right.
$$

gives him a payoff of value 3 . The weakness of this approach is that there is no theory of how to determine the set $\tilde{\Theta}$ and of how to construct the mechanism $\sigma_{1}$ for values of $\theta$ which are not in $\Theta$. Indeed, Martimort [22] characterizes the equilibrium using the Martimort-Stole algorithm.

\section{Conclusions}

Almost all of the literature on common agency with incomplete information focuses on equilibria that can be characterized by direct mechanisms. ${ }^{5}$ Some papers explicitly apply the Revelation Principle even if it is not applicable. By doing this they may characterize only a subset of all equilibria, and miss some realistic equilibria. Other articles use different mathematical tools and more complex mechanisms, but without characterizing a larger set of equilibria. We still lack a simple, general, systematic approach for characterizing all of the equilibria of a large class of common agency games. The complexity of the existing methodology (menu or extended types) indicates that this will be a demanding task.

\section{Acknowledgements}

I am grateful to Andrea Attar, Eloisa Campioni and Piero Gottardi for their comments and suggestions. I thank also François Boldron, Cyril Hariton, Sylvain Latil, Patrick Leoni and Martin Meier and seminar participants at CORE and at the European Economic Association Congress in Amsterdam for their help and comments. All errors are mine.

\section{References}

[1] M. Peters, "Common Agency and the Revelation Principle," Econometrica, Vol. 69, No. 5, September 2001, pp. $1349-1372$.

[2] D. Martimort and L. Stole, "The Revelation and Delegation Principles in Common Agency Games," Econometrica, Vol. 70, No. 4, July 2002, pp. 1659-1673.

[3] J.-J. Laffont and D. Martimort, "The Theory of Incentives," Princeton University Press, Princeton, 2002.

[4] P. Hammond, "Straightforward Individual Incentive Compatibility in Large Economies," Review of Economic Studies, Vol. 46, No. 2, April 1979, pp. 263-282.

[5] R. Guesnerie, "On Taxation and Incentives; Futher Re-

$\overline{{ }^{5} \text { The equilibrium characterized in Biais and Mariotti [12] is a notable }}$ exception. 
marks on the Limits to Redistribution," Bonn Discussion Paper, 1981.

[6] J.-C. Rochet, "Le contrôle des équations aux dérivées partielles issues de la théorie des incitations," $\mathrm{PhD}$ thesis, Université Paris IX, 1986.

[7] J.-J. Laffont and J. Tirole, "A Theory of Incentives in Procurement and Regulation," MIT Press, Cambridge, 1993.

[8] J. Peck, "A Note on Competing Mechanisms and the Revelation Principle," Mimeo, Ohio State University, 1997. http://economics.sbs.ohio-state.edu/jpeck/reveal.pdf

[9] M. Peters, "Negotiation and Take-It or Leave-It in Common Agency," Journal of Economic Theory, Vol. 111, No. 1, July 2003, pp. 88-109.

[10] M. Hellwig, "On Moral Hazard and Non-Price Equilibria in Competitive Insurance Markets," Discussion Paper No. 109, Sonderforschungsbereich 21, Bonn, May 1983.

[11] A. Bisin, P. Gottardi and D. Guaitoli, "A Note on the Convergence to Competitive Equilibria in Economies with Moral Hazard," In: P. J. J. Herings, G. Van der Laan, and A. J. J. Talman, Eds., Theory of Markets, North Holland, 1999, pp. 229-246.

[12] B. Biais and T. Mariotti, "Strategic Liquidity Supply and Security Design," Review of Economic Studies, Vol. 72, No. 8, July 2005, pp. 615-649.

[13] D. Martimort, "Exclusive Dealing, Common Agency and Multiprincipal Incentives Theory," RAND Journal of Economics, Vol. 27, No. 1, Spring 1996, pp. 1-31.
[14] B. Biais, D. Martimort and J.-C. Rochet, "Competing Mechanisms in a Common Value Environment," Econometrica, Vol. 78, No. 4, July 2000, pp. 799-837.

[15] G. Calzolari, "Incentive Regulation of Multinational Enterprises," International Economic Review, Vol. 45, No. 1, February 2004, pp. 257-282.

[16] J.-J. Laffont and J. Pouyet, "The Subsidiarity Bias in Regulation," Journal of Public Economics, Vol. 88, No. 1, January 2004, pp. 255-283.

[17] D. Martimort and L. Stole, "Contractual Externalities and Common Agency Equilibria," Advances in Theoretical Economics, Vol. 3, No. 1, 2003. http://www.bepress.com/ bejte/advances/Vol3/iss1/art4

[18] F. Khalil, D. Martimort and B. Parigi, "Monitoring a Common Agent," Journal of Economic Theory, Vol. 135, No. 1, July 2007, pp. 35-67.

[19] T. Olsen and P. Osmundsen, "Multinationals, Regulatory Competition and Outside Options," Mimeo, Norwegian School of Economics and Business Administration, 2003.

[20] K. Diaw and J. Pouyet, "The Dilemma of Tax Competition: How (Not) to Attract (Inefficient) Frms?" CentER Discussion Paper No. 2004-68, 2004.

[21] G. Calzolari and C. Scarpa, "Non-Intrinsic Common Agency," ENI-FEEM Nota di lavoro 84.99, 2004. http:// ssrn.com/abstract $=200558$

[22] D. Martimort, "Multi-Principaux Avec Anti-Selection," Annales d'Economie et de Statistique, Vol. 28, No. 1, October 1992, pp. 1-37. 